\title{
Universal Scattering of Ultracold Atoms and Molecules in Optical Potentials
}

\author{
Hui Li ${ }^{1, *}$, Ming Li ${ }^{1}$, Constantinos Makrides ${ }^{2}$, Alexander Petrov ${ }^{1,3}$ and Svetlana Kotochigova ${ }^{1}$ \\ 1 Department of Physics, Temple University, Philadelphia, PA 19122, USA; tug38145@temple.edu (M.L.); \\ alexsandernp@gmail.com (A.P.); skotoch@temple.edu (S.K.) \\ 2 Joint Quantum Institute, National Institute of Standards and Technology, and University of Maryland, \\ Gaithersburg, MD 20899, USA; makrides@umd.edu \\ 3 Department of Physics, Saint Petersburg State University, Ulyanovskaya 3, Saint Petersburg 198504, Russia \\ * Correspondence: tuh40763@temple.edu
}

Received: 29 January 2019; Accepted: 12 March 2019; Published: 15 March 2019

check for updates

\begin{abstract}
Universal collisions describe the reaction of molecules and atoms as dominated by longrange interparticle interactions. Here, we calculate the universal inelastic rate coefficients for a large group of ultracold polar molecules in their lower ro-vibrational states colliding with one of their constituent atoms. The rate coefficients are solely determined by values of the dispersion coefficient and reduced mass of the collisional system. We use the $a b$ initio coupled-cluster linear response method to compute dynamic molecular polarizabilities and obtain the dispersion coefficients for some of the collisional partners and use values from the literature for others. Our polarizability calculations agree well with available experimental measurements. Comparison of our inelastic rate coefficients with results of numerically exact quantum-mechanical calculations leads us to conjecture that collisions with heavier atoms can be expected to be more universal.
\end{abstract}

Keywords: ultracold atom-molecule collisions; chemical reactions; universal model; dispersion interaction; van der Waals coefficients; dynamic polorizability

\section{Introduction}

Recent creation of nearly quantum-degenerate gases of polar $\mathrm{KRb}[1], \mathrm{RbCs}[2,3], \mathrm{NaK}[4,5]$, $\mathrm{NaRb}$ [6], and $\mathrm{NaLi}$ [7] molecules have opened up ultracold-controlled physics and chemistry research. The first four of these alkali-metal molecules were formed in their absolute ground singlet $\mathrm{X}^{1} \Sigma^{+}$state, while NaLi was prepared in the energetically-lowest triplet a ${ }^{3} \Sigma^{+}$state. Since these molecules were created in an optical trap they can collide among each other or with residual ultracold atoms and undergo chemical reactions at sub- $\mu \mathrm{K}$ temperatures [8-11]. Quantum threshold phenomena then control the collision outcome, where a single collisional partial wave $\vec{\ell}$ dominates. Often, this is the zero relative orbital angular momentum corresponding to a collision with no entrance centrifugal barrier. We note that collisions among the alkali-metal dimers $\mathrm{KRb}$ or $\mathrm{NaLi}$ are reactive regardless of their ro-vibrational state. For the other three dimers, the molecule must be vibrationally excited [12] for the reaction to occur.

The first measurement of the reaction rate coefficient between ultracold $\mathrm{KRb}$ molecules and $\mathrm{K}$ atoms was made at JILA [8]. A schematic of this reaction is shown in Figure 1. The atom-molecule reaction rate coefficient is surprisingly high on the order of $10^{-10} \mathrm{~cm}^{3} / \mathrm{s}$ even at temperatures below $1 \mu \mathrm{K}$. Quantum defect theory (QDT) calculations [13-15] showed that the reaction is nearly universal and suggests that the attractive long-range van-der-Waals interaction between the neutral particles plays a prominent role. It is worth noting that the ${ }^{40} \mathrm{~K}^{87} \mathrm{Rb}$ isotopologue in Reference [8] is a fermion and when prepared in a single quantum state, $\mathrm{KRb}+\mathrm{KRb}$ collisions are dominated by $\ell=1$, 
$p$-wave collisions at ultracold temperatures. For fermionic molecules, $s$-wave collisions can only occur when they are prepared in different internal states (i.e., electronic, vibrational, rotational, and/or nuclear). Elastic resonant scattering between alkali-metal dimers and its heaviest constituent atom has been explored in Reference [16]. In fact, some Feshbach resonances have been observed in $500 \mathrm{nK}$ ${ }^{23} \mathrm{Na}^{40} \mathrm{~K}+{ }^{40} \mathrm{~K}$ collisions [17].

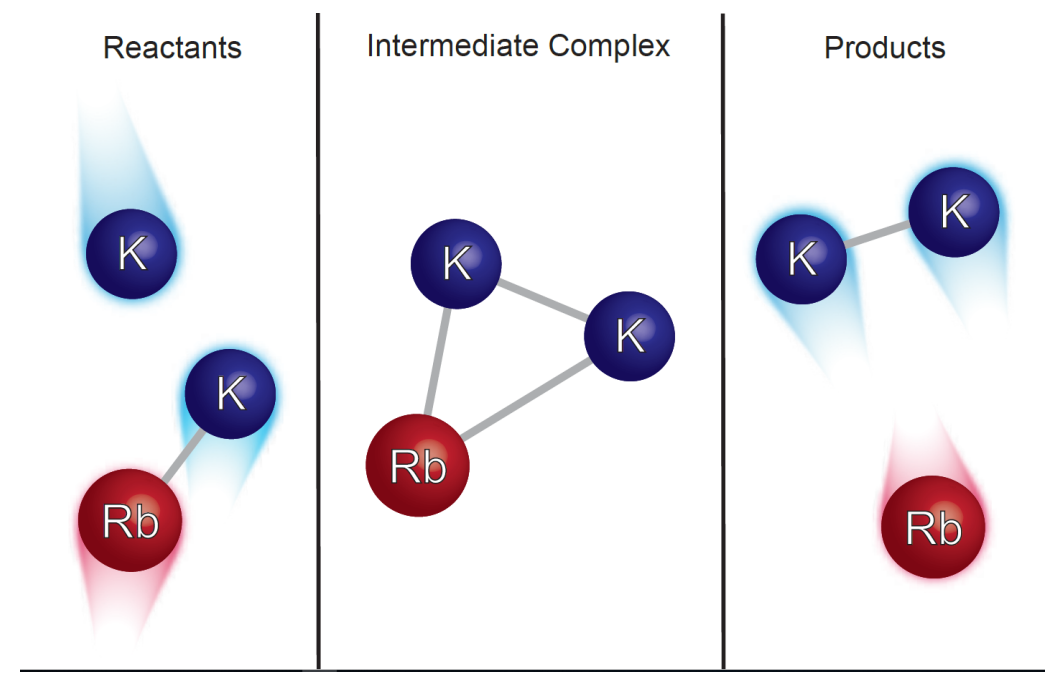

Figure 1. Possible reaction mechanisms for a colliding KRb molecule and a $\mathrm{K}$ atom. The chemical reaction proceeds via intermediate collisional complex to the final reaction products.

The $\mathrm{KRb}+\mathrm{K}$ and other reaction-rate measurements [18-22] have focused on total reaction rate coefficients. The next logical step in the field is to measure as well as calculate final-state resolved distributions. Such detailed understanding of the ultracold reaction might require a rigorous quantum treatment. Initial examples are the theoretical simulations of the state-to-state reaction rate for $\mathrm{LiYb}+\mathrm{Li}$ and $\mathrm{KRb}+\mathrm{K}$ using a numerically-exact quantum-mechanical (EQM) method formulated in hyperspherical coordinates $[23,24]$. These simulations led to a better understanding of the reaction mechanism and product ro-vibrational distribution and might also guide the development of experimental state-selective detection tools.

Total reaction rate coefficients for ultracold collisions between alkali-metal atoms and molecules have been studied with quantum defect theories. The most important outcome was a thorough understanding of the non-classical scattering from the long-range van-der-Waals dispersion potential. Scattering when the atom and molecule are close together is summarized in terms of a few collision-energy- and partial-wave-independent parameters. They describe the amplitude and phase of flux returning from the chemical bonding region and are boundary conditions for quantum solutions or wavefunctions at larger particle separations. The special case where no flux returns is known as universal scattering. For rotation-less molecules, the van-der-Waals dispersion potential is isotropic. In a number of cases [23-25] these QDT results have been validated with comparisons with "exact" quantum simulations.

In Sections 2 and 3, we setup and analyze universal ultracold scattering calculations for collisions among many alkali-metal, alkaline-earth and rare-earth heteronuclear dimers and their constituent atoms. We calculate the reactive scattering or vibrational-quenching rate coefficients of these diatomic molecules when prepared in ro-vibrational state $v, j$, assuming universal scattering $[13,14,26]$. Here, $v$ and $j$ are the vibrational and rotational quantum numbers of the diatomic molecule, respectively. In particular, we only consider collisions with rotation-less $j=0$ molecules. For the $v=0, j=0$ state, only reactive processes are available. We compare the rate coefficients with experimental measurements or exact numerical calculations, where available. If not specifically mentioned, the isotopes ${ }^{6} \mathrm{Li},{ }^{23} \mathrm{Na}$, ${ }^{40} \mathrm{~K},{ }^{87} \mathrm{Rb},{ }^{87} \mathrm{Sr},{ }^{133} \mathrm{Cs}$, and ${ }^{174} \mathrm{Yb}$ are implied. 
The most-relevant property for universal scattering is the isotropic dispersion coefficient $C_{6}^{\text {iso }}$ between atoms and molecules. It is determined by an integral over imaginary frequency of a product of atomic and molecular dynamic polarizabilities. In Section 4 we compute molecular polarizabilities based on coupled-cluster response theory using the time evolution of linear response functions and compute the isotropic $C_{6}^{\text {iso }}$. For several systems we use the isotropic dispersion coefficient of References [14,23,27]. We conclude in Section 5.

\section{Quantum Defect Theory and the Universal Model}

In this section, we highlight some important aspects of the universal model (UM) taken from Reference [14]. The UM is a quantum defect model for the reaction or vibrational quenching of rotation-less $j=0^{2 S+1} \Sigma^{+}$diatomic molecules colliding with S-state atoms under ultracold conditions. The theory is a modification of the approach developed in References [28,29] and has been successful in qualitatively and sometimes quantitatively describing observed ultracold reaction rate coefficients. In this model, we assume that the long-range interaction between a molecule and an atom is an attractive isotropic $-C_{6}^{\text {iso }} / R^{6}$ van-der-Waals potential, with characteristic length $R_{6}=\left(2 \mu C_{6}^{\text {iso }} / \hbar^{2}\right)^{1 / 4}$ and energy scale $\mathcal{E}_{6}=\hbar^{2} /\left(2 \mu R_{6}^{2}\right)$. Here, $R$ is the separation between the atom and the center of mass of the molecule, $\mu$ is the reduced mass of the system, and $\hbar$ is the reduced Planck constant. (For molecules with $j>0$ the dispersion potential has anisotropic contributions. We do not treat these cases here).

The collision for a molecule in vibrational state $v$ can then be described by the radial Schrödinger equation

$$
\left(-\frac{\hbar^{2}}{2 \mu} \frac{d^{2}}{d R^{2}}+\frac{\hbar^{2}}{2 \mu} \frac{\ell(\ell+1)}{R^{2}}-\frac{C_{6}^{\text {iso }}}{R^{6}}\right) \psi_{\ell}(R)=E \psi_{\ell}(R),
$$

where $E$ is the relative collision energy, $\ell$ is the relative orbital angular momentum quantum number, and $\psi_{\ell}(E)$ is the radial wavefunction. Equation (1) is applicable for $R>R_{\mathrm{sr}}$, where separation $R_{\mathrm{sr}}$ is defined by $C_{6}^{\text {iso }} / R_{\mathrm{sr}}^{6} \equiv 2 B_{v}$, where $B_{v}$ is the rotational constant of vibrational state $v$. The rotational constant is much larger than $\mathcal{E}_{6}$ and thus $R_{\mathrm{sr}}<R_{6}$.

The short-range physics when $R<R_{\text {sr }}$ and all three atoms are close together is complex. Nevertheless, the effects of the short-range interaction can be summarized by the boundary condition

$$
\psi_{\ell}(R)=A\left(e^{+i\left[\left(R / R_{6}\right)^{-2} / 2-\pi / 4\right]}-Y_{\ell}(E) e^{-i\left[\left(R / R_{6}\right)^{-2} / 2-\pi / 4\right]}\right)
$$

at $R=R_{\mathrm{sr}}$, where $A$ is a normalization constant and $\gamma_{\ell}(E)=\zeta_{\ell}(E) e^{2 i \delta_{\ell}(E)}$ is the so-called short-range scattering amplitude. The real dimensionless parameters $\delta_{\ell}(E)$ and $\zeta_{\ell}(E) \in[0,1]$ represent the relative phase and amplitude of the flux returning from short range, respectively [14]. We then numerically propagate the wavefunction from $R_{\mathrm{sr}}$ to $R \gg R_{6}$ and fit

$$
\psi_{\ell}(R)=e^{-i(k R-\ell \pi / 2)}-S^{\ell}(E) e^{i(k R-\ell \pi / 2)},
$$

where $S^{\ell}(E)$ is the S-matrix element and wavenumber $k$ is defined through $E=\hbar^{2} k^{2} /(2 \mu)$.

In principle, $\delta_{\ell}(E)$ and $\zeta_{\ell}(E)$ depend on $E$ and $\ell$; however, quantum defect theory and our UM assumes that these dependences are weak or absent. The key feature of the UM is that when the colliding atom and molecule reach $R_{\mathrm{sr}}$ they will either react or quench. This equivalent to setting $\zeta_{\ell}(E)=0$ and $\delta_{\ell}(E)$ no longer appears in the short-range boundary condition. The observable total elastic and total reaction rate coefficients are

$$
K_{\text {elas }}(E)=v_{\mathrm{r}} \frac{\pi}{k^{2}} \sum_{\ell=0}^{\infty}(2 \ell+1)\left|1-S_{\text {elas }}^{\ell}(E)\right|^{2}
$$


and

$$
K_{\mathrm{loss}}(E)=v_{\mathrm{r}} \frac{\pi}{k^{2}} \sum_{\ell=0}^{\infty}(2 \ell+1)\left(1-\left|S_{\mathrm{elas}}^{\ell}(E)\right|^{2}\right)
$$

respectively, where $v_{\mathrm{r}}=\hbar k / \mu$ is the relative velocity. At ultracold temperatures, only a few partial waves $\ell$ contribute, as for higher $\ell$ the centrifugal barrier prevents the particles from approaching each other and high- $\ell$ contributions to $K_{\text {elas }}(E)$ and $K_{\text {loss }}(E)$ are negligibly small.

\section{Comparisons of Inelastic Rate Coefficients for Atom-Molecule Collisions}

In this section we discuss the elastic as well as reaction or quenching rate coefficients between an S-state atom and the lower ro-vibrational states of a $X^{1} \Sigma^{+}$or $X^{2} \Sigma^{+}$molecule based on the universal model, where incoming scattering flux penetrating to short-range is not reflected back, as well as compare its predictions to the Langevin model [30] and EQM calculations based on full potential surfaces, where available. To be precise, we focus on the reactive processes $\mathrm{AM}\left(\mathrm{M}^{\prime}\right)\left(\mathrm{X}^{2 S+1} \Sigma^{+}, v=0, j=0\right)+\mathrm{A} \rightarrow \mathrm{A}_{2}+\mathrm{M}\left(\mathrm{M}^{\prime}\right)$ and $\mathrm{AM}\left(\mathrm{X}^{2 S+1} \Sigma^{+}, v>0, j=0\right)+$ $\mathrm{A} \rightarrow \mathrm{AM}\left(\mathrm{X}^{2 S+1} \Sigma^{+}, v^{\prime}, j^{\prime}\right)+\mathrm{A}$ for non-reactive systems, where atoms $\mathrm{A}$ and $\mathrm{M}\left(\mathrm{M}^{\prime}\right)$ are distinct, atom $\mathrm{A}$ is an alkali-metal atom, $\mathrm{M}$ is an alkali-metal atom, and $\mathrm{M}^{\prime}$ is either alkaline-earth atom $\mathrm{Sr}$ or rare-earth atom $\mathrm{Yb}$. Moreover, for quenching processes the energy of ro-vibrational state $v^{\prime}, j^{\prime}$ is less than that of pair $v, j$. The $C_{6}^{\text {iso }}$ dispersion coefficients for our model are computed and discussed in the next section, Section 4 .

Figure 2 shows an example of the total elastic and reactive rate coefficient for $\mathrm{LiNa}+\mathrm{Li}$ collisions based on the universal model as function of collision energies up to $E=k_{\mathrm{B}} \times 1 \mathrm{~K}$. For collision energies below $k_{\mathrm{B}} \times 0.1 \mathrm{mK}$ only $\ell=0$, s-wave collisions contribute. In fact, the loss rate coefficient is independent of $E$, while the elastic rate coefficient is proportional to $\sqrt{E}$, i.e., the collision is said to be in the Wigner-threshold regime. For $E>k_{\mathrm{B}} \times 0.1 \mathrm{mK} p$-, $d$-, and higher partial-wave contributions become significant. Moreover, for $K_{\text {loss }}(E)$ the sum of these partial wave contributions approaches the loss rate coefficient based on the Langevin model with $\left|S^{\ell}(E)\right|^{2}=0$ or 1 when the collision energy is smaller or larger than the centrifugal barrier for the potential $-C_{6}^{\text {iso }} / R^{6}+\hbar^{2} \ell(\ell+1) /\left(2 \mu R^{2}\right)$. For dispersive long-range potentials, the Langevin model gives $K_{\text {loss }}(E) \propto E^{1 / 6}$.

Figure 3 shows total inelastic reactive or quenching rate coefficients based on the universal model as functions of collision energy for various $\mathrm{AM}\left(\mathrm{M}^{\prime}\right)+\mathrm{A}$ atom-molecule collisions. We observe that $\mathrm{AM}\left(\mathrm{M}^{\prime}\right)+\mathrm{A}$ systems are exothermic from $v=0$ when the mass of atom $\mathrm{A}$ is less than that of atom $\mathrm{M}\left(\mathrm{M}^{\prime}\right)$ and endothermic otherwise. For the endothermic systems we assume that the molecule is prepared in a vibrational state with small $v>0$ and present quenching rate coefficients. For small $v$, the isotropic dispersion coefficient is still well approximated by that for $v=0$. (This simple mass-based rule for reactivity does not always hold. Generally, the molecular dissociation energies of reactants and products must be compared [12]. For our choices of $\mathrm{A}$ and $\mathrm{M}\left(\mathrm{M}^{\prime}\right)$, which mainly belong to the first column of the periodic table, the rule does hold as dissociation energies are smaller for heavier molecules).

Exact quantum-mechanical results for $\mathrm{KRb}+\mathrm{K}$ and $\mathrm{LiYb}+\mathrm{Li}$ with molecules in the $\mathrm{X}^{1} \Sigma^{+}$and $\mathrm{X}^{2} \Sigma^{+}$potentials, respectively, have been published in References [23,24], respectively. They are compared to the s-wave contribution to the rate coefficient predicted by the universal model as well as its summed, total value in Figure 4. The EQM rate coefficients have only been computed for $s$-wave collisions as such calculations for other partial waves are currently numerically intractable. From the figure it is clear that $K_{\text {loss }}(E)$ values from the EQM and universal model agree for collision energies above $k_{\mathrm{B}} \times 1 \mathrm{mK}$ for both reactions. Below $k_{\mathrm{B}} \times 0.1 \mathrm{mK}$ the two calculations do not agree for $\mathrm{LiYb}+\mathrm{Li}$ with its small reduced mass. For the heavier $\mathrm{KRb}+\mathrm{K}$ collisions, the agreement remains excellent. In other words, for sub-100 $\mu \mathrm{K} \mathrm{LiYb}+\mathrm{Li}$ collisions there is a significant probability that flux is returned from the short-range region interferes with the incoming flux. Hence, we conclude that the 
full potential surface, including its short-range shape, used in the EQM calculations is important or that the reduced mass of the colliding partners plays a larger role than expected.

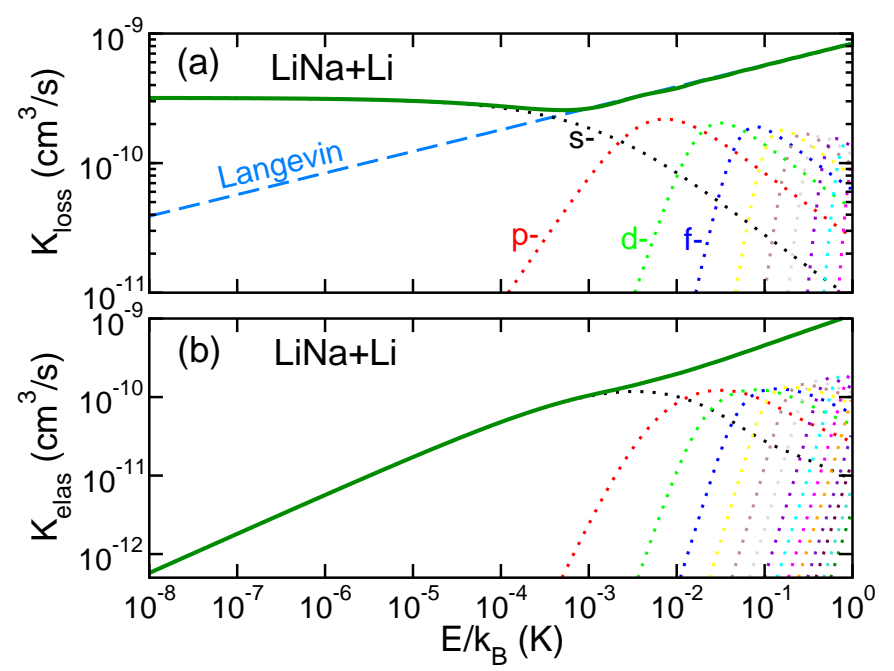

Figure 2. LiNa + Li total reaction (panel (a)) and elastic (panel (b)) rate coefficient for the universal model as a function of collision energy. The LiNa dimer is prepared in its ro-vibrational $\mathrm{X}^{1} \Sigma^{+}$ground state. $\mathrm{Li}_{2}$ product molecules are energetically-allowed ro-vibrational states of the $\mathrm{X}^{1} \Sigma_{g}^{+}$potential. Dotted lines correspond to the individual partial-wave contributions to the rate coefficients. In panel (a) the reaction rate coefficient based on the Langevin model is shown by the long-dashed blue line. Finally, $k_{\mathrm{B}}$ is the Boltzmann constant.
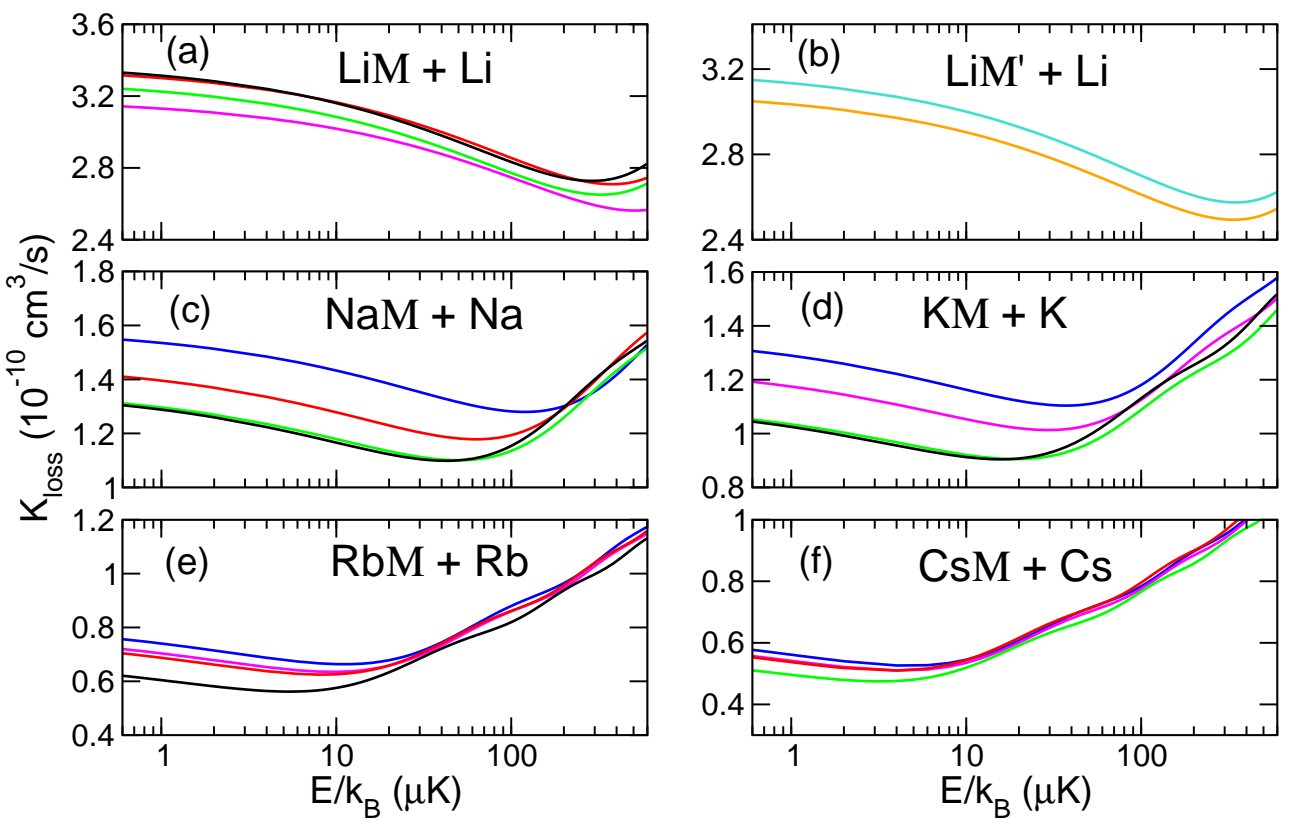

Figure 3. Inelastic reaction or quenching rate coefficients for alkali-metal, Strontium and Ytterbium atom-molecule collisions as functions of collision energy based on the universal model. The six panels show rate coefficients for the $\mathrm{AM}\left(\mathrm{M}^{\prime}\right)+\mathrm{A}$ collision, where $\mathrm{A}$ is either $\mathrm{Li}, \mathrm{Na}, \mathrm{K}, \mathrm{Rb}$, or Cs. In panels $(\mathbf{a}, \mathbf{c}-\mathbf{f})$ blue, dark green, red, green, and black curves correspond to rate coefficients for atom $\mathrm{M}=\mathrm{Li}, \mathrm{Na}, \mathrm{K}, \mathrm{Rb}$, and $\mathrm{Cs}$, respectively. In panel $(\mathbf{b})$ cyan and orange curves correspond to $\mathrm{M}^{\prime}=\mathrm{Sr}$ and $\mathrm{Yb}$, respectively. The molecules are prepared in their $\mathrm{X}^{1} \Sigma^{+}$ground state for alkali-metal dimers and in their $\mathrm{X}^{2} \Sigma^{+}$ground state for alkali-metal $\mathrm{Sr}$ or alkali-metal $\mathrm{Yb}$ dimers. Scattering is only reactive when the mass of atom $A$ is less than that of $M\left(M^{\prime}\right)$. When the mass of $A$ is larger than that of $M\left(M^{\prime}\right)$, quenching rate coefficients are presented. 


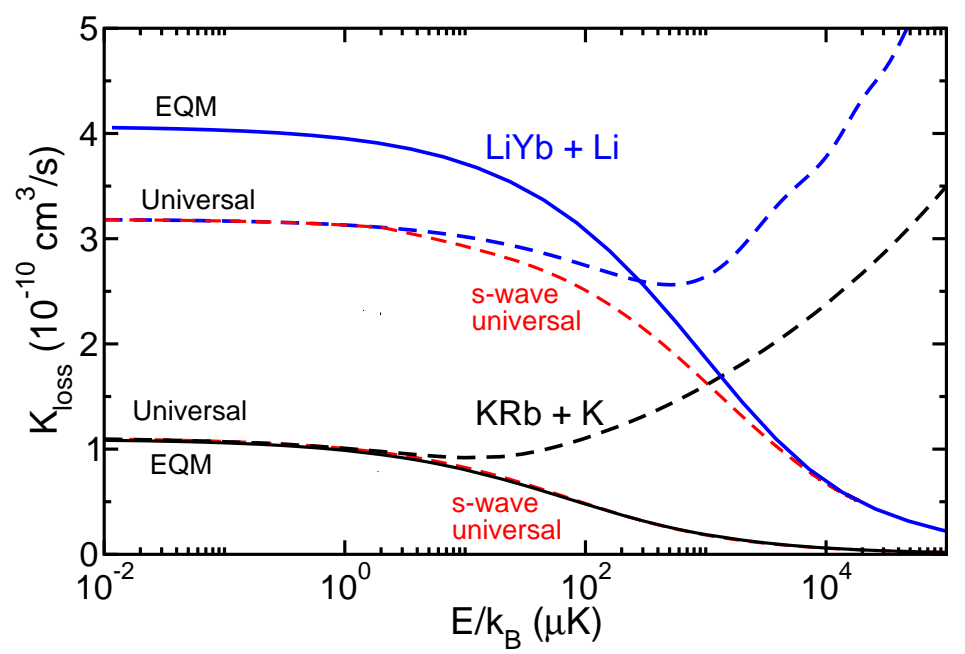

Figure 4. Reaction rate coefficients for $\mathrm{LiYb}\left(\mathrm{X}^{2} \Sigma^{+}, v=0, j=0\right)+\mathrm{Li}$ and $\mathrm{KRb}\left(\mathrm{X}^{1} \Sigma^{+}, v=0, j=0\right)+\mathrm{K}$ based on the universal model (dashed lines) and $s$-wave, $\ell=0$ exact quantum coupled-channels simulations based on a full potential energy surface (solid lines) as functions of collision energy. Red and blue dashed curves for $\mathrm{LiYb}+\mathrm{Li}$ collisions correspond to the s-wave contribution to $K_{\mathrm{loss}}(E)$ and the total $K_{\text {loss }}(E)$ based on the universal model, respectively. Red and black dashed curves are corresponding $K_{\text {loss }}(E)$ for $\mathrm{KRb}+\mathrm{K}$ collisions The EQM rate coefficients are from References [23,24].

\section{Molecular Dynamic Polarizability and Van Der Waals Coefficients}

In this section, we calculate some of the molecular dynamic polarizabilities of heteronuclear dimers for both real and imaginary frequencies and the isotropic atom-dimer van-der-Waals coefficients used in the previous section. Following Reference [31] and in atomic units the isotropic dispersion coefficient for the long-range interaction between a $j=0^{2 S+1} \Sigma^{+}$molecule and a ground $S$-state atom is

$$
C_{6}^{\text {iso }}=\frac{3}{\pi} \int_{0}^{\infty} \bar{\alpha}^{\mathrm{Mol}}(i v) \bar{\alpha}^{\mathrm{At}}(i v) d v,
$$

where $\bar{\alpha}^{\mathrm{Mol}}(i v)$ and $\bar{\alpha}^{\mathrm{At}}(i v)$ at imaginary frequency iv are mean molecular and atomic polarizabilities in atomic units, respectively, defined as $\bar{\alpha}=\left(\alpha_{x x}+\alpha_{y y}+\alpha_{z z}\right) / 3$ in terms of the diagonal tensor elements $\alpha_{n n}$ along the spatial coordinate axes $n=x, y$, and $z$. For $S$-state atoms $\alpha_{x x}=\alpha_{y y}=\alpha_{z z}$, while for di-atomic molecules $\alpha_{\perp} \equiv \alpha_{x x}=\alpha_{y y}$ is the parallel polarizability and $\alpha_{\|} \equiv \alpha_{z z}$ is the perpendicular polarizability in a body-fixed coordinate frame with the $z$ coordinate along the internuclear axis. For atomic dynamic polarizabilities as functions of imaginary frequency, we use the results in Reference [32].

The dynamic polarizability of ground-state heteronuclear dimers has two contributions. We write $\bar{\alpha}^{\mathrm{Mol}}(v)=\bar{\alpha}_{\text {ind }}^{\mathrm{Mol}}(v)+\bar{\alpha}_{\text {pol }}^{\mathrm{Mol}}(v)$, where $\bar{\alpha}_{\text {ind }}^{\mathrm{Mol}}(v)$ is the induction contribution from electric dipole transitions to ro-vibrational states within the ground X potential and $\bar{\alpha}_{\mathrm{pol}}^{\mathrm{Mol}}(v)$ is the polarization contribution from transitions to all other electronic states. The former contribution is due to the finite permanent electronic dipole moment of a heteronuclear dimer, denoted by $d_{j}(R)$ as a function of $R$ and electronic state $j$. Similarly, we have $C_{6}^{\text {iso }}=C_{6, \text { ind }}^{\text {iso }}+C_{6, \text { pol }}^{\text {iso }}$. In 2010, Reference [14] already showed that this induction contribution to $C_{6}^{\text {iso }}$ is important for $\mathrm{RbCs}$ but not $\mathrm{KRb}$, due to the large permanent dipole moment of RbCs.

In principle, the mean molecular dynamic polarizability as a function of imaginary frequency depends on the ro-vibrational level of the ground state electronic molecule. For small $v$ and $j=0$ the vibrational wavefunctions are localized near the equilibrium separation $R_{e}$. For the purposes of evaluating dispersion coefficients it is then sufficient to evaluate $\bar{\alpha}^{\mathrm{Mol}}(i v)$ at $R=R_{e}$. (The approximation is invalid when $\bar{\alpha}^{\mathrm{Mol}}(v)$ at real frequencies nearly resonant between ro-vibrational 
states of ground and electronic potentials are needed). The mean induction component for $j=0$ ${ }^{2 S+1} \Sigma^{+}$molecules at imaginary frequencies is then to good approximation

$$
\bar{\alpha}_{\text {ind }}^{\mathrm{Mol}}(i v)=\frac{1}{3} d_{\mathrm{X}}^{2}\left(R_{e}\right) \frac{4 B_{e}}{\left(2 B_{e}\right)^{2}+v^{2}}
$$

in atomic units, where $d_{\mathrm{X}}\left(R_{e}\right)$ is the permanent dipole moment of the molecular ground $\mathrm{X}$ state at $R_{e}$ and $B_{e}$ is the rotational constant at this separation. Moreover, as the atomic polarizability is essentially independent of $v$ between $\mu=0$ and a few times $B_{e}$, the induction contribution to the isotropic van der Waals coefficient becomes $C_{6, \text { ind }}^{\text {iso }}=d_{\mathbf{X}}^{2}\left(R_{e}\right) \alpha(0)$, where $\alpha(0)$ is the (static) dynamic polarizability of the atom at zero frequency $[14,27]$.

We have calculated the polarization contributions $\alpha_{\|, \text {pol }}^{\mathrm{Mol}}\left(v, R_{e}\right)$ and $\alpha_{\perp, \text { pol }}^{\mathrm{Mol}}\left(v, R_{e}\right)$ as functions of real frequency $v$ at $R=R_{e}$ with coupled-cluster linear-response (CCLR) theory for a selected set of molecules. Others were available in the literature. The CCLR method was developed by Monkhorst and Dalgaard [33,34], generalized by Koch and Jørgensen [35], and used to derive expressions for the polarization contribution to the molecular polarizability as a function of real frequencies and nuclear positions [36]. We use the implementation as coded in CFOUR, an electronic structure software package [37], with single and double excitations [38]. The all-electron cc-pCVQZ basis set of the Peterson group [39] is used for $\mathrm{Li}$ and $\mathrm{Na}$ in calculation of polarizability of the LiNa molecule. For KRb and $\mathrm{RbCs}$ molecules we combine the relativistic effective-core potential ECP10MDF, ECP28MDF, and ECP46MDF from Reference [40] with the basis sets def2-TZVPP $(17 \mathrm{~s}, 11 \mathrm{p}, 3 \mathrm{~d}) /[6 \mathrm{~s}, 4 \mathrm{p}, 3 \mathrm{~d}]$ for K, (7s7p5d1f)/[6s4p3d1f] for Rb, and (7s6p3d1f)/[5s3p3d1f] for Cs from Reference [41], respectively. For LiSr molecule we used the basis set of def2-QZVPP (15s6p2d1f)/[6s4p2d1f] for Li, and def2-QZVPP (8s8p5d3f)/[7s5p4d3f] combined with ECP28MDF for Sr.

The calculated polarization contributions are then fit to the resonant form

$$
\alpha_{n, \mathrm{pol}}^{\mathrm{Mol}}\left(v, R_{e}\right)=\sum_{j} \frac{f_{n, j}}{1-\left(v / \eta_{n, j}\right)^{2}}
$$

with parameters $f_{n, j}$ and $\eta_{n, j}$ for $n=\|$ and $\perp$ following the microscopic definition of the polarizability in terms of a sum over excited electronic states and resonant energy denominators. Hence, $f_{n, j}$ and $\eta_{n, j}$ are related to the oscillator strength and transition frequency between the ground state and excited state $j$ at $R=R_{e}$, respectively. Finally, the polarizability at imaginary frequency follows from the substitution $v \rightarrow i v$ and the polarization contribution to the isotropic $C_{6}^{\text {iso }}$ from numerical integration of Equation (6). Table 1 lists all $C_{6}^{\text {iso }}$ used in this paper as well as gives references to the values not computed by us.

Table 1. Isotropic $C_{6}^{\text {iso }}$ dispersion coefficients in atomic units for various atom-molecule systems at their

\begin{tabular}{|c|c|c|c|c|c|c|c|c|c|}
\hline $\mathrm{LiNa}+\mathrm{Li}$ & 2112 & $\mathrm{NaLi}+\mathrm{Na}$ & 2241 & KLi + K & $5341^{a}$ & $\mathrm{RbLi}+\mathrm{Rb}$ & $6478^{a}$ & $\mathrm{CsLi}+\mathrm{Cs}$ & $9455^{\mathrm{a}}$ \\
\hline $\mathrm{LiK}+\mathrm{Li}$ & $3179^{a}$ & $\mathrm{NaK}+\mathrm{Na}$ & $3592^{a}$ & $\mathrm{KNa}+\mathrm{K}$ & $5698^{a}$ & $\mathrm{RbNa}+\mathrm{Rb}$ & $6896^{a}$ & $\mathrm{CsNa}+\mathrm{Cs}$ & $9879^{a}$ \\
\hline $\mathrm{LiRb}+\mathrm{Li}$ & $3505^{a}$ & $\mathrm{NaRb}+\mathrm{Na}$ & $3948^{a}$ & $\mathrm{KRb}+\mathrm{K}$ & $6905^{c}$ & $\mathrm{RbK}+\mathrm{Rb}$ & $7696^{c}$ & $\mathrm{CsK}+\mathrm{Cs}$ & $11,237^{a}$ \\
\hline $\mathrm{LiCs}+\mathrm{Li}$ & $4192^{\mathrm{a}}$ & $\mathrm{NaCs}+\mathrm{Na}$ & $4631^{\mathrm{a}}$ & $\mathrm{KCs}+\mathrm{K}$ & $8473^{a}$ & $\mathrm{RbCs}+\mathrm{Rb}$ & 9506 & $\mathrm{CsRb}+\mathrm{Cs}$ & 11,414 \\
\hline $\mathrm{LiSr}+\mathrm{Li}$ & 3137 & & & & & & & & \\
\hline $\mathrm{LiYb}+\mathrm{Li}$ & $3086^{b}$ & & & & & & & & \\
\hline
\end{tabular}
equilibrium separation. Values without label $a, b$, or $c$ have been computed specifically for this paper.

Figure 5 shows two examples of the polarization contribution to the molecular polarizability as functions of real frequency calculated with the CCLR method at $R=R_{e}$. Parallel, perpendicular and isotropic components have been computed for frequencies in the visible and infrared region. For KRb, the static $(v \rightarrow 0)$ limit is also shown. Each curve has no more than three resonances, corresponding to 
the $\mathrm{X}^{1} \Sigma^{+}$to $\mathrm{A}^{1} \Sigma^{+}$transition for the parallel component. Those for the perpendicular component are due to transitions to the two energetically-lowest ${ }^{1} \Pi$ potentials.

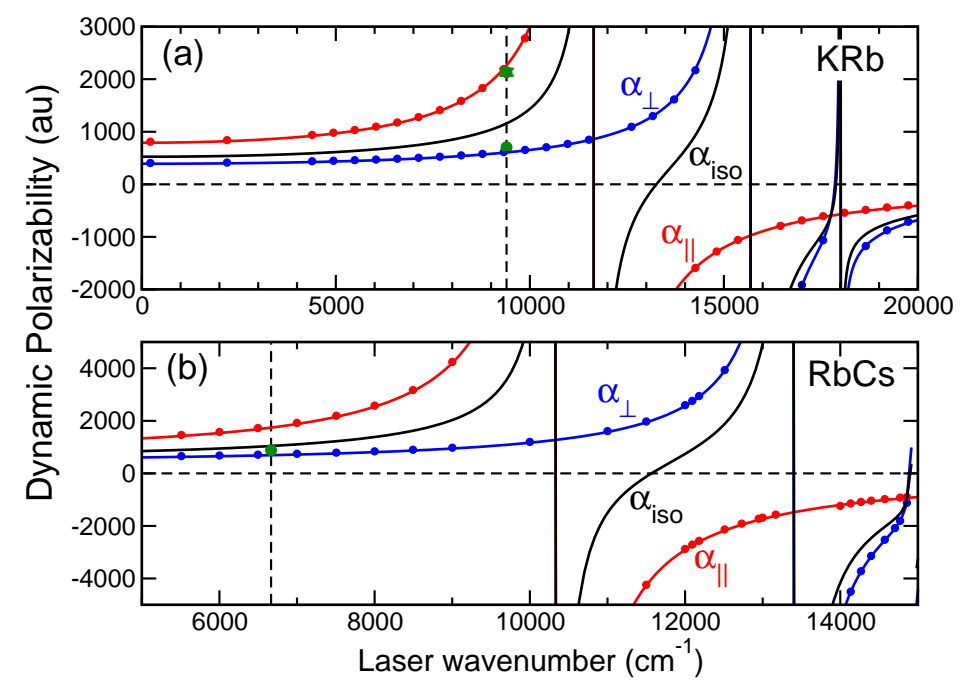

Figure 5. The CCLR polarization contribution to the molecular dynamic polarizability of the $\mathrm{X}^{1} \Sigma^{+}$ ground state of $\mathrm{KRb}(\mathbf{a})$ and $\mathrm{RbCs}(\mathbf{b})$ at their equilibrium separation in atomic units as functions of laser wavenumber. Red, blue, and black curves and markers correspond to the parallel $\alpha_{\|}$, perpendicular $\alpha_{\perp}$, and isotropic $\bar{\alpha}=\alpha_{\text {iso }}$ dynamic polarizability, respectively. Markers represent frequencies on which the polarizabilities have been computed; curves are the fits. In panel (a) the dark green markers near $9300 \mathrm{~cm}^{-1}$ represent the experimental results from Reference [42], while in panel (b) the marker near $6667 \mathrm{~cm}^{-1}$ represents the experimental results from Reference [43].

\section{Conclusions}

Our calculation of universal reactive or quenching rate coefficients for a large group of alkali-metal and alkaline-earth molecules colliding with one of their constituent atoms has shown that the inelastic rates are large on the order of a few times $10^{-10} \mathrm{~cm}^{3} / \mathrm{s}$ corresponding to gas lifetimes of a fraction of a second for number densities on the order of $10^{10} \mathrm{~cm}^{-3}$. The rate coefficients are minimal near collision energies of $k_{\mathrm{B}} \times 0.5 \mathrm{mK}$ for collisions with $\mathrm{Li}$ atoms. For collisions between heavier particles this minimum shifts to lower collision energies. In addition, inelastic rate coefficients are smaller for systems with a larger reduced mass.

In addition, we find a clear agreement between EQM reaction-rate and the $s$-wave contribution to the universal value for collision energies above $k_{\mathrm{B}} \times 1 \mathrm{mK}$. Below $k_{\mathrm{B}} \times 0.1 \mathrm{mK}$, however, the two calculations disagree for $\mathrm{LiYb}+\mathrm{Li}$. This implies that for such low collision energies there exists a significant probability that flux returned from the short-range region interferes with the incoming flux. On the other hand, the excellent agreement between the two $\mathrm{KRb}+\mathrm{K}$ calculations for this Wignerthreshold scattering indicates that all flux that reaches the short range reacts.

We have also compared ab initio CCLR dynamic polarizabilities for $\mathrm{KRb}$ and $\mathrm{RbCs}$ with accurate experimental measurements of References [42,43] and found excellent agreement. These results serve as evidence of quality of our coupled-cluster CCLR calculations.

Finally, we look at possible future research directions. The $\mathrm{KRb}+\mathrm{K}$ loss rate coefficient has been measured [8] and is twice as large as the UM prediction. In Reference [17] Feshbach resonances have been observed in the $\mathrm{NaK}+\mathrm{K}$ collision through the observation of magnetic-field-dependent loss rate coefficients. Within the quantum defect theory we might be able to describe this dependence by relaxing the requirement of a $100 \%$ short-range absorption probability as well as assuming a magneticfield-dependent short-range amplitude. Early research on this subject can be found in Reference [14]. 
Author Contributions: All authors contributed to carrying out the theoretical investigation and interpretation of the results. H.L. and S.K. also contributed to writing the manuscript.

Funding: Work at the Temple University is supported by the Army Research Office Grant No. W911NF-17-1-0563, the U.S. Air Force Office of Scientific Research Grant No. FA9550-14-1-0321 and the NSF Grant No. PHY-1619788.

Conflicts of Interest: The authors declare no conflict of interest.

\section{References}

1. Ni, K.K.; Ospelkaus, S.; de Miranda, M.H.G.; Pe'er, A.; Neyenhuis, B.; Zirbel, J.J.; Kotochigova, S.; Julienne, P.S.; Jin, D.S.; Ye, J. A High Phase-Space-Density Gas of Polar Molecules. Science 2008, 322, $231-235$. [CrossRef] [PubMed]

2. Takekoshi, T.; Reichsöllner, L.; Schindewolf, A.; Hutson, J.M.; Le Sueur, C.R.; Dulieu, O.; Ferlaino, F.; Grimm, R.; Nägerl, H.C. Ultracold Dense Samples of Dipolar RbCs Molecules in the Rovibrational and Hyperfine Ground State. Phys. Rev. Lett. 2014, 113, 205301. [CrossRef]

3. Molony, P.K.; Gregory, P.D.; Ji, Z.; Lu, B.; Köppinger, M.P.; Le Sueur, C.R.; Blackley, C.L.; Hutson, J.M.; Cornish, S.L. Creation of Ultracold ${ }^{87} \mathrm{Rb}^{133} \mathrm{Cs}$ Molecules in the Rovibrational Ground State. Phys. Rev. Lett. 2014, 113, 255301. [CrossRef] [PubMed]

4. Park, J.W.; Will, S.A.; Zwierlein, M.W. Ultracold Dipolar Gas of Fermionic ${ }^{23} \mathrm{Na}^{40} \mathrm{~K}$ Molecules in Their Absolute Ground State. Phys. Rev. Lett. 2015, 114, 205302. [CrossRef] [PubMed]

5. Seeßelberg, F.; Buchheim, N.; Lu, Z.K.; Schneider, T.; Luo, X.Y.; Tiemann, E.; Bloch, I.; Gohle, C. Modeling the adiabatic creation of ultracold polar ${ }^{23} \mathrm{Na}^{40} \mathrm{~K}$ molecules. Phys. Rev. A 2018, 97, 013405. [CrossRef]

6. Guo, M.; Zhu, B.; Lu, B.; Ye, X.; Wang, F.; Vexiau, R.; Bouloufa-Maafa, N.; Quéméner, G.; Dulieu, O.; Wang, D. Creation of an Ultracold Gas of Ground-State Dipolar ${ }^{23} \mathrm{Na}^{87} \mathrm{Rb}$ Molecules. Phys. Rev. Lett. 2016, 116, 205303. [CrossRef] [PubMed]

7. Rvachov, T.M.; Son, H.; Sommer, A.T.; Ebadi, S.; Park, J.J.; Zwierlein, M.W.; Ketterle, W.; Jamison, A.O. Long-Lived Ultracold Molecules with Electric and Magnetic Dipole Moments. Phys. Rev. Lett. 2017, 119, 143001. [CrossRef] [PubMed]

8. Ospelkaus, S.; Ni, K.K.; Wang, D.; de Miranda, M.H.G.; Neyenhuis, B.; Quéméner, G.; Julienne, P.S.; Bohn, J.L.; Jin, D.S.; Ye, J. Quantum-State Controlled Chemical Reactions of Ultracold Potassium-Rubidium Molecules. Science 2010, 327, 853-857. [CrossRef] [PubMed]

9. de Miranda, M.H.G.; Chotia, A.; Neyenhuis, B.; Wang, D.; Quéméner, G.; Ospelkaus, S.; Bohn, J.; Ye, J.; Jin, D. Controlling the quantum stereodynamics of ultracold bimolecular reactions. Nat. Phys. 2011, 7, 502. [CrossRef]

10. Julienne, P.S.; Hanna, T.M.; Idziaszek, Z. Universal ultracold collision rates for polar molecules of two alkali-metal atoms. Phys. Chem. Chem. Phys. 2011, 13, 19114-19124. [CrossRef] [PubMed]

11. Quéméner, G.; Julienne, P.S. Ultracold molecules under control! Chem. Rev. 2012, 112, 4949-5011. [CrossRef]

12. Zuchowski, P.; Hutson, J.M. Reactions of ultracold alkali-metal dimers. Phys. Rev. A 2010, 81, 060703(R). [CrossRef]

13. Idziaszek, Z.; Julienne, P.S. Universal Rate Constants for Reactive Collisions of Ultracold Molecules. Phys. Rev. Lett. 2010, 104, 113202. [CrossRef]

14. Kotochigova, S. Dispersion interactions and reactive collisions of ultracold polar molecules. New J. Phys. 2010, 12, 073041. [CrossRef]

15. Quéméner, G.; Bohn, J.L.; Petrov, A.; Kotochigova, S. Universalities in ultracold reactions of alkali-metal polar molecules. Phys. Rev. A 2011, 84, 062703. [CrossRef]

16. Mayle, M.; Ruzic, B.P.; Bohn, J.L. Statistical aspects of ultracold resonant scattering. Phys. Rev. A 2012, 85, 062712. [CrossRef]

17. Yang, H.; Zhang, D.C.; Liu, L.; Liu, Y.X.; Nan, J.; Zhao, B.; Pan, J.W. Observation of magnetically tunable Feshbach resonances in ultracold ${ }^{23} \mathrm{Na}^{40} \mathrm{~K}+{ }^{40} \mathrm{~K}$ collisions. Science 2019, 363, 261-264. [CrossRef] [PubMed]

18. Hudson, E.R.; Gilfoy, N.B.; Kotochigova, S.; Sage, J.M.; DeMille, D. Inelastic Collisions of Ultracold Heteronuclear Molecules in an Optical Trap. Phys. Rev. Lett. 2008, 100, 203201. [CrossRef] 
19. Deiglmayr, J.; Repp, M.; Wester, R.; Dulieu, O.; Weidemüller, M. Inelastic collisions of ultracold polar LiCs molecules with caesium atoms in an optical dipole trap. Phys. Chem. Chem. Phys. 2011, 13, 19101-19105. [CrossRef]

20. De Marco, L.; Valtolina, G.; Matsuda, K.; Tobias, W.G.; Covey, J.P.; Ye, J. A degenerate Fermi gas of polar molecules. Science 2019. [CrossRef]

21. Li, Z.; Gong, T.; Ji, Z.; Zhao, Y.; Xiao, L.; Jia, S. A dynamical process of optically trapped singlet ground state ${ }^{85} \mathrm{Rb}^{133} \mathrm{Cs}$ molecules produced via short-range photoassociation. Phys. Chem. Chem. Phys. 2018, 20, 4893-4900. [CrossRef]

22. Ye, X.; Guo, M.; González-Martínez, M.L.; Quéméner, G.; Wang, D. Collisions of ultracold ${ }^{23} \mathrm{Na}^{87} \mathrm{Rb}$ molecules with controlled chemical reactivities. Sci. Adv. 2018, 4. [CrossRef]

23. Makrides, C.; Hazra, J.; Pradhan, G.B.; Petrov, A.; Kendrick, B.K.; González-Lezana, T.; Balakrishnan, N.; Kotochigova, S. Ultracold chemistry with alkali-metal-rare-earth molecules. Phys. Rev. A 2015, 91, 012708. [CrossRef]

24. Croft, J.; Makrides, C.; Li, M.; Petrov, A.; Kendrick, B.; Balakrishnan, N.; Kotochigova, S. Universality and chaoticity in ultracold $\mathrm{K}+\mathrm{KRb}$ chemical reactions. Nat. Commun. 2017, 8, 15897. [CrossRef]

25. Frye, M.D.; Julienne, P.S.; Hutson, J.M. Cold atomic and molecular collisions: Approaching the universal loss regime. New J. Phys. 2015, 17, 045019. [CrossRef]

26. Gao, B. Universal Model for Exoergic Bimolecular Reactions and Inelastic Processes. Phys. Rev. Lett. 2010, 105, 263203. [CrossRef]

27. Żuchowski, P.S.; Kosicki, M.; Kodrycka, M.; Soldán, P. van der Waals coefficients for systems with ultracold polar alkali-metal molecules. Phys. Rev. A 2013, 87, 022706. [CrossRef]

28. Julienne, P.S.; Mies, F.H. Collisions of ultracold trapped atoms. J. Opt. Soc. Am. B 1989, 6, $2257-2269$. [CrossRef]

29. Orzel, C.; Walhout, M.; Sterr, U.; Julienne, P.S.; Rolston, S.L. Spin polarization and quantum-statistical effects in ultracold ionizing collisions. Phys. Rev. A 1999, 59, 1926-1935. [CrossRef]

30. Quéméner, G.; Honvault, P.; Launay, J.M.; Soldán, P.; Potter, D.E.; Hutson, J.M. Ultracold quantum dynamics: Spin-polarized $\mathrm{K}+\mathrm{K}_{2}$ collisions with three identical bosons or fermions. Phys. Rev. A 2005, 71, 032722. [CrossRef]

31. Stone, A.J. The Theory of Intermolecular Forces; Clarendon Press: London, UK, 1996.

32. Derevianko, A.; Porsev, S.G.; Babb, J.F. Electric dipole polarizabilities at imaginary frequencies for hydrogen, the alkali-metal, alkaline-earth, and noble gas atoms. At. Data Nucl. Data Tables 2010, 96, 323-331. [CrossRef]

33. Monkhorst, H.J. Calculation of properties with the coupled-cluster method. Int. J. Quantum Chem. 1997, 12, 421-432. [CrossRef]

34. Dalgaard, E.; Monkhorst, H.J. Some aspects of the time-dependent coupled-cluster approach to dynamic response functions. Phys. Rev. A 1983, 28, 1217-1222. [CrossRef]

35. Koch, H.; Jörgensen, P. Coupled cluster response functions. J. Chem. Phys. 1990, 93, 3333-3344. [CrossRef]

36. Kobayashi, R.; Koch, H.; Jörgensen, P. Calculation of frequency-dependent polarizabilities using coupledcluster response theory. Chem. Phys. Lett. 1994, 219, 30-35. [CrossRef]

37. Stanton, J.F.; Gauss, J.; Harding, M.E.; Szalay, P.G. Computer Code CFOUR: A Program Package to Perform High-Level Quantum Chemical Calculations on Atoms and Molecules; University of Texas: Austin, TX, USA, 2011.

38. Watts, J.D.; Gauss, J.; Bartlett, R.J. Coupled-cluster methods with noniterative triple excitations for restricted open-shell Hartree-Fock and other general single determinant reference functions. Energies and analytical gradients. J. Chem. Phys. 1993, 98, 8718. [CrossRef]

39. Prascher, B.P.; Woon, D.E.; Peterson, K.A.; Dunning, T.H.; Wilson, A.K. Gaussian basis sets for use in correlated molecular calculations. VII. Valence, core-valence, and scalar relativistic basis sets for $\mathrm{Li}, \mathrm{Be}, \mathrm{Na}$, and Mg. Theor. Chem. Acc. 2011, 128, 69-82. [CrossRef]

40. Lim, I.S.; Schwerdtfeger, P.; Metz, B.; Stoll, H. All-electron and relativistic pseudopotential studies for the group 1 element polarizabilities from K to element 119. J. Chem. Phys. 2005, 122, 104103. [CrossRef]

41. Weigend, F.; Ahlrichs, R. Balanced basis sets of split valence, triple zeta valence and quadruple zeta valence quality for H to Rn: Design and assessment of accuracy. Phys. Chem. Chem. Phys. 2005, 7, 3297-3305. [CrossRef] 
42. Neyenhuis, B.; Yan, B.; Moses, S.A.; Covey, J.P.; Chotia, A.; Petrov, A.; Kotochigova, S.; Ye, J.; Jin, D.S. Anisotropic Polarizability of Ultracold Polar ${ }^{40} \mathrm{~K}^{87} \mathrm{Rb}$ Molecules. Phys. Rev. Lett. 2012, 109, 230403. [CrossRef]

43. Gregory, P.D.; Blackmore, J.A.; Aldegunde, J.; Hutson, J.M.; Cornish, S.L. ac Stark effect in ultracold polar ${ }^{87} \mathrm{Rb}^{133}$ Cs molecules. Phys. Rev. A 2017, 96, 021402. [CrossRef]

(C) 2019 by the authors. Licensee MDPI, Basel, Switzerland. This article is an open access article distributed under the terms and conditions of the Creative Commons Attribution (CC BY) license (http://creativecommons.org/licenses/by/4.0/). 